\title{
Phenomenological Aspects of Quantum Gravity and Modified Theories of Gravity
}

\author{
Ahmed Farag Ali, ${ }^{1,2}$ Giulia Gubitosi, ${ }^{3}$ Mir Faizal, ${ }^{4}$ and Barun Majumder ${ }^{5}$ \\ ${ }^{1}$ Benha University, Benha, Egypt \\ ${ }^{2}$ Netherlands Institute for Advanced Study, Korte Spinhuissteeg 3, 1012 CG Amsterdam, Netherlands \\ ${ }^{3}$ Imperial College London, London, UK \\ ${ }^{4}$ British Columbia University, Vancouver, BC, Canada \\ ${ }^{5}$ Indian Institute of Technology-Gandhinagar, Gujarat, India
}

Correspondence should be addressed to Ahmed Farag Ali; ahmed.ali@fsc.bu.edu.eg

Received 12 December 2016; Accepted 20 December 2016; Published 15 February 2017

Copyright ( 2017 Ahmed Farag Ali et al. This is an open access article distributed under the Creative Commons Attribution License, which permits unrestricted use, distribution, and reproduction in any medium, provided the original work is properly cited. The publication of this article was funded by SCOAP S $^{3}$

The phenomenological aspects of various approaches to quantum gravity and modified theories of gravity are getting a lot of attention and cover many different topics such as theories with minimal length, noncommutative geometry, theories with generalized uncertainty principle, violations of Lorentz symmetries, and loop quantum cosmology. The recent developments in these approaches with their phenomenological implications in particle physics, cosmology, and astrophysics are the main theme of this special issue. In this special issue on quantum gravity phenomenology, we have invited papers that address such issues.

In the paper by S. Dutta et al., the authors present a study on emergent universe scenario with modified Chaplygin gas and its implications with the initial big bang singularity. In the paper by P. Pedram, the author uses the modified commutation relation, which has quadratic order in the momentum as deformation, to study the Beckner, Bialynicki-Birula, and Mycielski (BBM) inequality. With a particular choice of self-adjoint representation, the author has shown that the BBM inequality remains valid for various choices of the deformation parameter. In the paper by $\mathrm{H}$. Moradpour and R. Dehghani, the authors tackled the unified first law of thermodynamics and its genuine connection with the apparent horizon of FRW universe in which they found that whenever there is no energy exchange between the various parts of cosmos, one could get an expression for the apparent horizon entropy in quasi-topological gravity. In the paper by S.-Z. Yang et al., the authors studied the Hawking radiation in the context of Lorentz invariance violation which has been predicted in different approaches to quantum gravity. In the paper by M. J. Soleimani et al, the authors investigate tunneling of charged massive particles in charged $\mathrm{TeV}$-scale black hole with the help of a generalized uncertainty relation that has a minimal length and maximal momentum. In the paper by $\mathrm{M}$. Ronco, the author tackled Planck-scale dynamical dimensional reduction in which he observed that the number of UV dimensions can be used to constrain the ambiguities in the choice of these loop quantum gravity-based modifications of the Dirac space-time algebra. In the paper by T. Moon and P. Oh, the authors studied spontaneous symmetry breaking in 5D conformally invariant gravity and found that the dimensional reduction via ADM decomposition gives rise to the $4 \mathrm{D}$ Minkowski vacuum. In the paper by Z.-W. Feng et al., the authors studied the implications of minimal length theories on the entropic force realization of gravity and they derived, in this context, the modified Einstein field equation and modified Friedman equations which may have various phenomenological implications. In the paper by A. E. Bernardini and R. Rocha, the authors have studied a family of asymmetric thick brane configurations which are formed by defects. A left-right asymmetric chiral localization of spin $1 / 2$ particles is produced in the localization of fermion fields of 
the thick branes. In the paper by S.-Z. Yang et al., the authors investigated the origin of Hawking radiation in the context of fermions tunneling from higher-dimensional ReissnerNordström black hole and calculated the entropy of the black hole. In the paper by F. Ghobakhloo and H. Hassanabadi, the authors investigated the free particle propagator in the context of minimal length theories and discussed possible implications of this modified propagator.

Ahmed Farag Ali Giulia Gubitosi Mir Faizal

Barun Majumder 

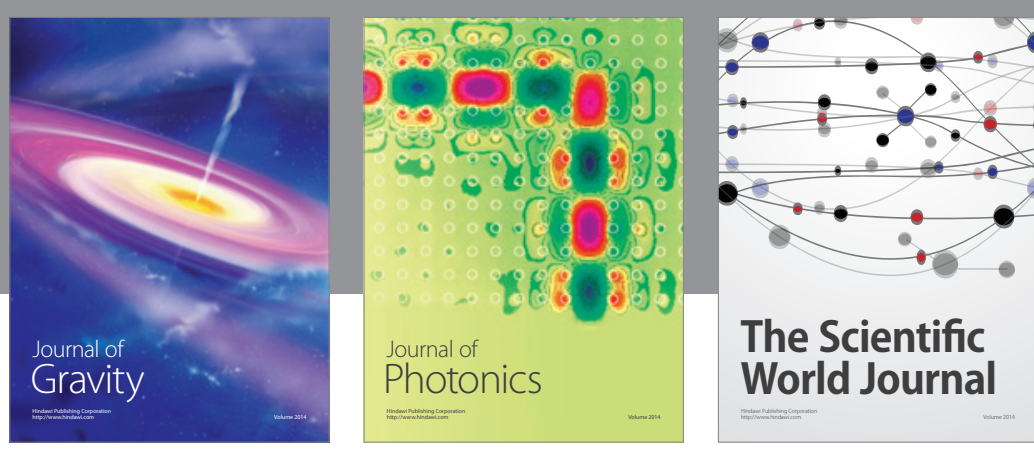

The Scientific World Journal
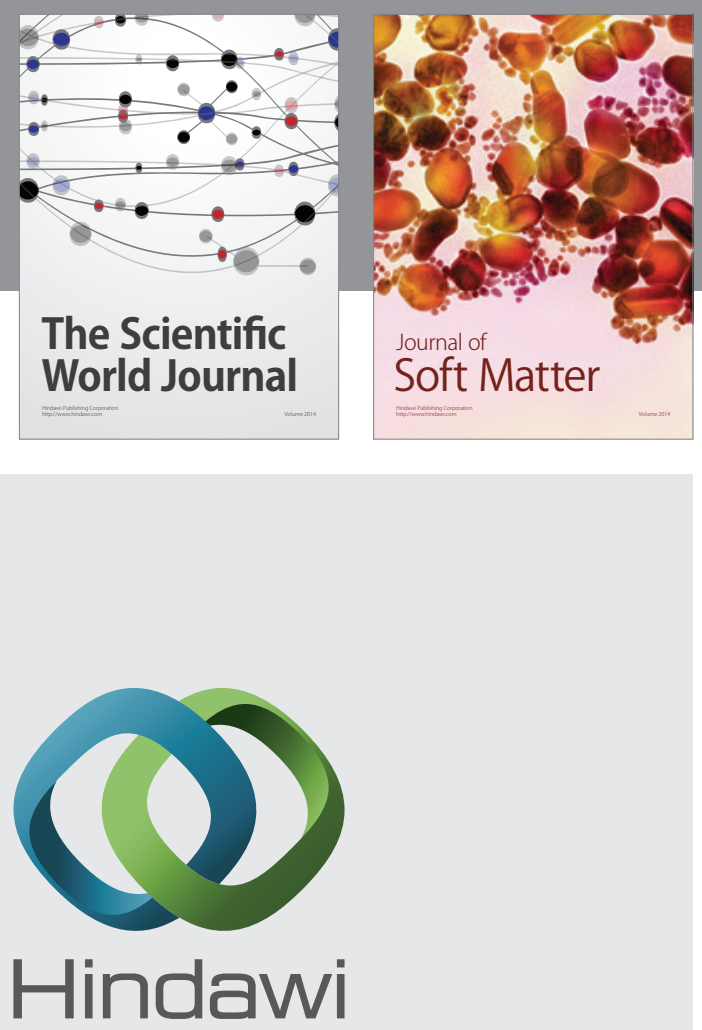

Submit your manuscripts at

https://www.hindawi.com
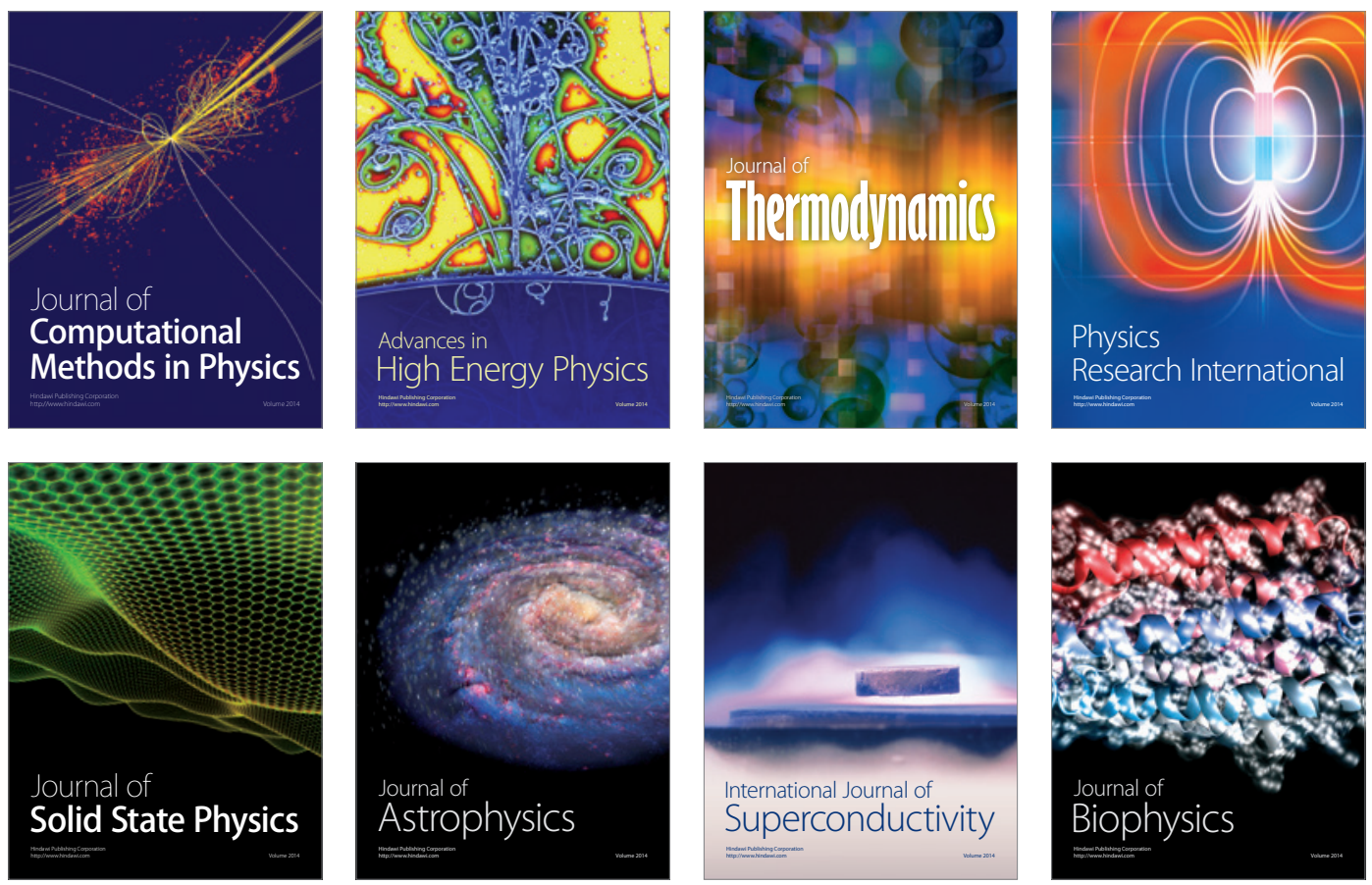
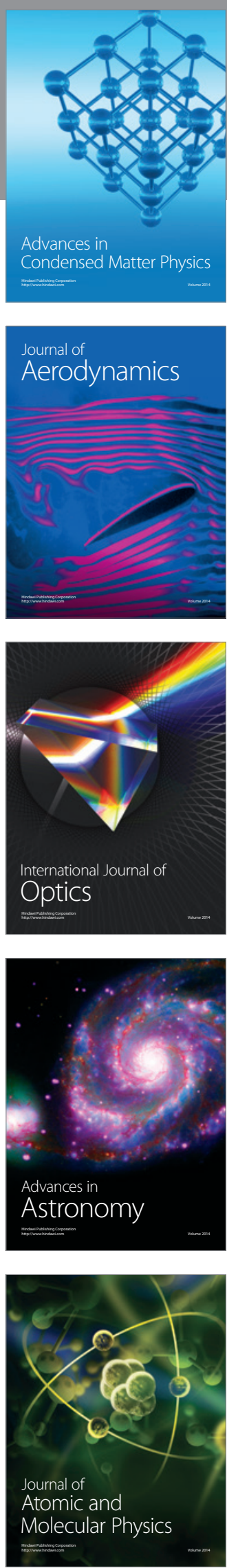